\title{
Evaluating the potential of respiratory- sinus-arrhythmia biofeedback for reducing physiological stress in adolescents with autism: study protocol for a randomized controlled trial
}

\author{
Anoushka Thoen ${ }^{1,2^{*}}$ (D) Jean Steyaert ${ }^{2,3}$ (D) Kaat Alaerts ${ }^{2,4}$ (i) and Tine Van Damme Ka $^{1,2,3}$
}

\begin{abstract}
Background: Prior evidence points towards lower cardiac vagal modulation in individuals with autism spectrum disorder (ASD) as compared to control groups. A cross-sectional phase in this study will gather more evidence concerning this topic. A longitudinal phase will explore the efficacy of a biofeedback intervention based on respiratory sinus arrhythmia (RSA) in adolescents with ASD. Finally, a feasibility study will focus on a non-supervised RSA biofeedback intervention in this population.

Methods: The cross-sectional phase includes the comparison of adolescents with ASD ( $n=38)$ and age- and gender-matched typically developing peers. A standardized assessment will be used which contains physiological, cortisol, and behavioral measurements. The longitudinal phase contains a randomized, single-blinded, and shamcontrolled design to determine the efficacy of supervised RSA biofeedback in adolescents with ASD $(n=128)$. A follow-up phase of 5 weeks is included to evaluate the presence of retention effects. During the latter, a feasibility study will focus on a non-supervised intervention $(n=64)$. Assessments as described previously are scheduled after the intervention and the follow-up phase.
\end{abstract}

Discussion: First, more conclusive evidence will be provided for the presence of lower cardiac vagal modulation in adolescents with ASD as well as the association between these lower values and physiological and behavioral indices. Second, the supervised intervention in adolescents with ASD is hypothesized to upregulate this cardiac vagal modulation and positively change behavioral and physiological parameters. Third, evidence regarding the feasibility and acceptability of a non-supervised intervention may open novel avenues for home-based interventions in this population.

Trial registration: ClinicalTrials.gov NCT04628715. Registered on 13 November 2020.

Keywords: Autism spectrum disorder, Cardiac vagal modulation, RSA biofeedback, Randomized controlled trial

\footnotetext{
* Correspondence: anoushka.thoen@kuleuven.be

'Department of Rehabilitation Sciences, Research Group for Adapted Physical

Activity and Psychomotor Rehabilitation, KU Leuven, Leuven, Belgium

${ }^{2}$ Leuven Autism Research (LAuRes), KU Leuven, Leuven, Belgium

Full list of author information is available at the end of the article
}

(c) The Author(s). 2021 Open Access This article is licensed under a Creative Commons Attribution 4.0 International License, which permits use, sharing, adaptation, distribution and reproduction in any medium or format, as long as you give appropriate credit to the original author(s) and the source, provide a link to the Creative Commons licence, and indicate if changes were made. The images or other third party material in this article are included in the article's Creative Commons licence, unless indicated otherwise in a credit line to the material. If material is not included in the article's Creative Commons licence and your intended use is not permitted by statutory regulation or exceeds the permitted use, you will need to obtain permission directly from the copyright holder. To view a copy of this licence, visit http://creativecommons.org/licenses/by/4.0/. The Creative Commons Public Domain Dedication waiver (http://creativecommons.org/publicdomain/zero/1.0/) applies to the data made available in this article, unless otherwise stated in a credit line to the data. 


\section{Background}

In the last decade, research concerning stressmechanisms in individuals with autism spectrum disorder (ASD) has increased exponentially. This neurodevelopmental disorder is characterized by problems in social interaction and communication and the presence of repetitive, stereotyped behaviors, activities, and interests [1]. In addition, children and adolescents with ASD report higher rates of stress as compared to typically developing (TD) peers [2, 3]. Especially in the adolescent period, which is characterized by rapid changes across several developmental domains (e.g., social context, affect regulation, and behavior), individuals with ASD are hypothesized to be more vulnerable for stress-related difficulties [4].

Researchers recently explored the contributing role of the autonomic nervous system in psychiatric disorders, including ASD [5-9]. One of the most commonly adopted physiological parameters for assessing autonomic nervous system functioning in psychophysiological research is based on the assessment of heart rate variability (HRV). The heart's pacemaker is controlled by both the sympathetic and the parasympathetic nervous system, which results in the natural variation of the interval between heartbeats. One important component of HRV is respiratory sinus arrhythmia (RSA). This component is solely mediated by the parasympathetic subsystem through direct projections of the vagus nerve from the brain stem to the heart and is therefore also known as an index of cardiac vagal modulation. The term RSA is adopted since it defines the phenomenon of the relation between heart rate variability and the respiratory cycle [10]. This parameter is typically identified in the high-frequency component of HRV (HF-HRV), which ranges between 0.15 and $0.4 \mathrm{~Hz}$, and by the root mean square of successive differences (RMSSD) of the interbeat intervals [11].

The clinical importance of RSA has been reported in a variety of studies demonstrating higher RSA to be associated with higher cognitive abilities, effective social behavior, fewer internalizing symptoms (e.g., anxiety and depression), and appropriate emotion regulation [1214]. Moreover, lower RSA has been linked to conduct problems, trait hostility, anxiety disorders, and depression $[12,15,16]$. Another important aspect of RSA is its positive association with appropriate social behavior as described by Porges' polyvagal theory [17]. The latter theory describes a parasympathetically driven social engagement system, which promotes the activation of interconnected craniofacial nerves and their associated motor behaviors. This way, a calm state-as induced by a heightened parasympathetic drive-is associated with prosocial behaviors including the making of eye contact, appropriate vocalizations, and socially oriented head turning [18]. Recent research indicated that TD children and adolescents demonstrated more prosocial behavior when higher RSA values were present $[18,19]$.

With respect to children and adolescents with ASD, the majority of studies have demonstrated lower levels of RSA in individuals with ASD during various activities $[5-7,9]$. This was confirmed in a recent meta-analysis, where statistically significant lower RSA-values were found in individuals with ASD during baseline measurements, social stress tasks, social debriefing tasks, and cognitive tasks [20]. Lower RSA levels in children and adolescents with ASD have been associated with higher levels of autism-specific symptoms such as social problems, internalizing problems, anxiety, emotional control issues, more severe visual and/or auditory sensory problems, and repetitive and restrictive behaviors [5-7, 9]. However, given methodological differences, multiple researchers have recommended further research on ANS functioning in individuals with ASD and how it deviates from TD peers [8, 18-21]. Therefore, a cross-sectional phase is included in this study in which a standardized stress-provoking protocol will be used in adolescents with and without ASD.

Considering the prior observations of lower RSA levels in individuals with ASD, it would be of high clinical relevance to develop and validate an intervention that specifically targets the upregulation of RSA values-a measure of cardiac vagal modulation-in individuals with ASD [9]. Prior HRV-RSA biofeedback studies have shown that an increase of HRV was causally related to several positive effects on psychological parameters. These studies were performed in distinct clinical populations of all ages and variable medical and psychological conditions [22-29]. In this protocol, individuals practice to breathe at their resonant frequency, which can be found between 4.5 and 7 breaths per minute. Breathing at this frequency will maximize HRV oscillations due to the entrainment of two physiological mechanisms affecting HRV, namely the influence of the baroreflex and of the breathing pattern [13]. For more information concerning the hypothesized working mechanisms, see Lehrer and Gevirtz [28]. Only one recent study provided evidence of feasibility of a similar intervention in a small sample $(n=7)$ of children and adolescents (10-15 years) with ASD [30]. The current study will encompass a large-scale randomized-controlled trial, in which a representative sample of adolescents with ASD will undergo a supervised RSA-based biofeedback training to test its efficacy for enhancing RSA levels and positively changing associated behavioral symptoms and physiological parameters as determined at the cross-sectional phase. Finally, a feasibility study regarding a nonsupervised RSA-based biofeedback intervention will be implemented for half of the adolescents with ASD after the supervised intervention part (see Fig. 1). 


\section{Specific aims and hypotheses}

This study consists of a cross-sectional and a longitudinal phase, each containing one research objective. An overview of the complete study design is presented in Fig. 1 and Table 1.

The first objective relates to the cross-sectional phase in which adolescents with ASD $(n=38 ; 19$ boys, $13-18$ years) and TD peers will be recruited. A standardized stress-provoking assessment will be used to provide insight into differences in autonomic functioning between adolescents with ASD and their TD peers. The primary outcome measure is cardiac vagal modulation, which is hypothesized to be lower in adolescents with ASD. In addition, other physiological and behavioral parameters as well as cortisol measurements will be included as secondary outcome measures. These group comparisons will be highly informative to delineate indices of ANS functioning that are specifically implicated in
ASD and to evaluate whether aberrant physiology in ASD is associated with clinical and behavioral indices (e.g., predictive of increased social difficulties, repetitive behaviors, and anxiety) as reported previously.

The second objective relates to the longitudinal phase in which only adolescents with ASD $(n=128)$ will be included. In this phase, the efficacy of a 5-week supervised RSA biofeedback intervention will be assessed by means of a randomized, single-blinded, and sham-controlled superiority design. The intervention is hypothesized to lead to increments of cardiac vagal modulation as opposed to the control intervention. To determine whether the latter hypothesized increment can be maintained, a 5 -week follow-up phase will be included. Further, it is hypothesized that an increase in cardiac vagal modulation relates to changes in behavior (e.g., improvement in social behavior) and/or physiological changes but that these might occur at a later stage, thus after the follow-

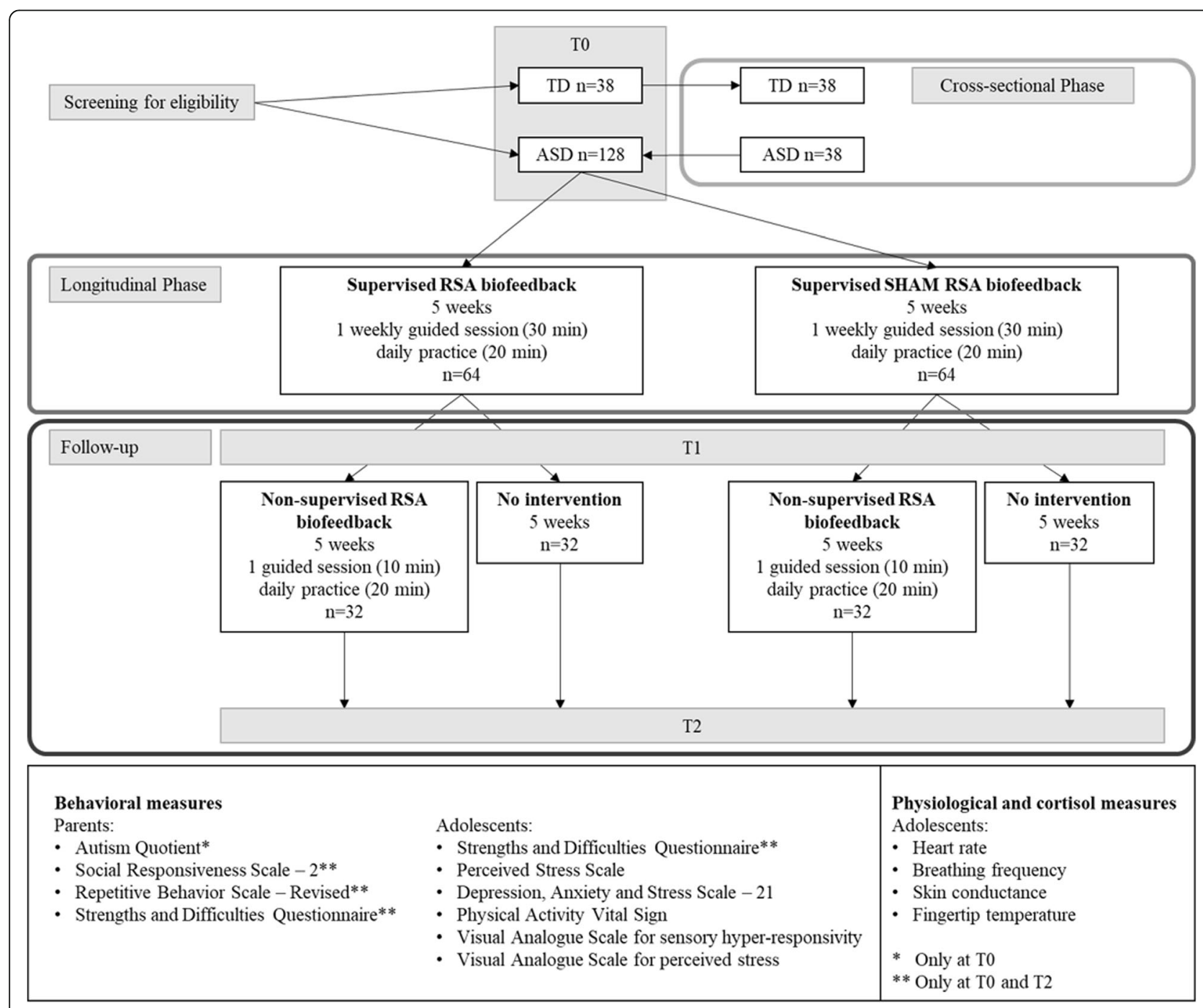

Fig. 1 Study flow diagram. Note: ASD, autism spectrum disorder; TD, typically developing; RSA, respiratory sinus arrhythmia 
Table 1 Procedure of enrolment, interventions, and assessment following SPIRIT guidelines. T0 baseline assessment, T1 assessment after 5 weeks of supervised intervention, T2 after 5 weeks of follow-up

\begin{tabular}{|c|c|c|c|c|c|c|c|c|}
\hline \multirow{2}{*}{\multicolumn{2}{|c|}{ Events }} & \multicolumn{2}{|c|}{$\begin{array}{c}\text { Cross-sectional } \\
\text { phase }\end{array}$} & \multicolumn{5}{|c|}{ Longitudinal phase } \\
\hline & & Enrolment & $\mathrm{T} 0$ & T0 & Allocation & $\mathrm{T} 1$ & $\begin{array}{l}\text { Follow- } \\
\text { up }\end{array}$ & $\mathrm{T} 2$ \\
\hline \multicolumn{2}{|l|}{ Informed consent } & $\mathrm{x}$ & & & & & & \\
\hline \multicolumn{2}{|l|}{ Eligibility screen } & $\mathrm{x}$ & & & & & & \\
\hline \multicolumn{2}{|c|}{ Randomization ASD } & & & $\mathrm{x}$ & & & $\mathrm{x}$ & \\
\hline \multirow{2}{*}{$\begin{array}{l}\text { Behavioral data } \\
\text { parents }^{\text {a }}\end{array}$} & $\mathrm{TD}$ & $\mathrm{x}$ & $\mathrm{x}$ & & & & & \\
\hline & ASD & & $\mathrm{x}$ & & & & & \\
\hline \multirow{2}{*}{$\begin{array}{l}\text { Behavioral data } \\
\text { adolescents }^{\mathrm{a}}\end{array}$} & $\mathrm{TD}$ & & $\mathrm{x}$ & & & & & \\
\hline & ASD & & $\begin{array}{c}x \\
(n=38)^{b}\end{array}$ & $\begin{array}{c}x \\
(n=90)\end{array}$ & $\begin{array}{c}x \\
(n=128)\end{array}$ & $\begin{array}{c}x \\
(n=128)\end{array}$ & & $\begin{array}{c}x \\
(n=128)\end{array}$ \\
\hline \multirow{2}{*}{$\begin{array}{l}\text { Physiological } \\
\text { and cortisol data } \\
\text { adolescents }\end{array}$} & TD & & $\mathrm{x}$ & & & & & \\
\hline & ASD & & $\begin{array}{c}x \\
(n=38)^{b}\end{array}$ & $\begin{array}{c}x \\
(n=90)\end{array}$ & $\begin{array}{c}x \\
(n=128)\end{array}$ & $\begin{array}{c}x \\
(n=128)\end{array}$ & & $\begin{array}{c}x \\
(n=128)\end{array}$ \\
\hline \multicolumn{2}{|c|}{$\begin{array}{l}\text { Supervised RSA } \\
\text { biofeedback intervention }\end{array}$} & & & & & 2 & & \\
\hline \multicolumn{2}{|c|}{$\begin{array}{l}\text { Feasibility study } \\
\text { non-supervised RSA } \\
\text { biofeedback } \\
\text { intervention }^{c}\end{array}$} & & & & & & & \\
\hline \multicolumn{6}{|c|}{$\begin{array}{l}\text { acquisition of these data is a prerequisite for the eligibility screen (Autism } \\
\text { Quotient data from parents of TD adolescents) and before physiological data } \\
\text { collection can take place (all behavioral data) } \\
\text { b Their selection is based on the sequence of enrollment and matching according to } \\
\text { age and gender with TD adolescents } \\
\text { c Performed by } 64 \text { adolescents with ASD; control-group ( } \mathrm{n}=64 \text { ) receives no } \\
\text { intervention }\end{array}$} & \multicolumn{3}{|c|}{$\begin{array}{l}\text { Note: ASD=Autism Spectrum } \\
\text { Disorder; TD=Typically } \\
\text { Developing; RSA=Respiratory } \\
\text { Sinus Arrhythmia }\end{array}$} \\
\hline
\end{tabular}

up phase. Therefore, other physiological and behavioral parameters as well as cortisol measurements will be assessed as well. In addition, a feasibility study concerning a non-supervised RSA biofeedback intervention will be performed by half of the participants $(n=64)$ during the follow-up phase.

\section{Methods}

The study is reported to conform to the SPIRIT (Standard Protocol Items: Recommendations for Interventional Trails) statement [31] (see Additional File 2 and 3).

\section{General procedure}

The study design is visualized in Table 1 and Fig. 1. During the cross-sectional phase, physiological, cortisol, and behavioral data will be gathered in adolescents with and without ASD, who are matched according to age and gender. This phase will also be used as a baseline measurement (T0) for the first 38 recruited and matched adolescents with ASD, who will subsequently be included in the longitudinal phase. Ninety additional adolescents with ASD will execute the same baseline measurement but their data will only be used for the longitudinal phase. After 5 weeks of supervised RSA-based biofeedback intervention (or sham-control), all adolescents with ASD $(n=128)$ will be re-assessed (T1). A follow-up session of 5 weeks will be included (T2) to assess the possibility of retention effects and to determine the presence of behavioral changes. During this follow-up phase, a feasibility study regarding a non-supervised RSA-based biofeedback intervention will be conducted by half of the adolescents with ASD $(n=64)$. All assessments and supervised sessions will be performed at a site from the KU Leuven or at the schools of the participants. In addition, the specific government regulations with regard to COVID-19 will be followed rigorously.

\section{Ethics approval and informed consent}

Ethical approval for this study was granted by both the Ethics Committee UPC KU Leuven on July 2, 2020 (ref. 
EC2020-541) and the Ethics Committee Research UZ/ KU Leuven on October 20, 2020 (ref: S64219) and on April 9, 2021 (ref: S64219, amendment). Parents of the participants should provide informed consent in addition to the informed assent provided by the participants themselves. The informed consent and assent forms will be gathered by AT. Participants may withdraw consent and participation at any time. The participant's request to withdraw from the study will always be respected and reasons to withdraw are not obliged to be mentioned. As the inclusion for the trial is based on the voluntary participation of the adolescents, no specific plans have been designed to promote the completion of the trial. However, the adolescents and their parents can contact the researchers at any time during the trial to express their concerns in order to find appropriate solutions; for instance with regard to planning the home-based sessions. The sponsor of this study is KU Leuven.

\section{Monitoring of side-effects}

An independent researcher, who is not involved in data analysis or data collection, will review potential reports of side effects for all participants during and after completion of the study and will be available to discuss side effects with the participants if needed. Standard operating procedures will be followed for reporting serious adverse events to researcher(s), the Ethical Committee, and applicable Competent Authorities (CA's) based on applicable legislation. All participants will be ensured through the no-fault policy of the KU Leuven.

\section{Participants}

A subgroup of adolescents with a formal diagnosis of ASD ( $n=38,19$ boys, $13-18$ years) based on DSM-IV/5 criteria [1,32] will participate in the cross-sectional phase while the total sample $(n=128 ; 64$ boys, $13-18$ years) will be included in the longitudinal phase of this study. An age- and gender-matched group of TD adolescents $(n=38)$ will be included in the cross-sectional phase only. Exclusion criteria are the presence of an intellectual disability as described in the DSM-IV/DSM-5; the presence of an uncorrected hearing or vision impairment; the presence of congenital heart diseases, diagnosed cardiovascular abnormalities or somatic diseases with a known impact on heart function; pregnancy; and insufficient knowledge of Dutch language and participation in other clinical trials. Care as usual can be continued. Use of medication and the presence of comorbid or co-occurring disorders will not lead to exclusion of the study but will be registered elaborately. For TD adolescents specifically, a score equal to or more than 32 on the Autism Quotient - Adolescent Version [33] and the presence of a neurodevelopmental disorder or psychiatric disorder as described in the DSM-IV/DSM-5 will lead to exclusion from the study. In addition, TD adolescents with a sibling diagnosed with a neurodevelopmental disorder as described in the DSM-IV/DSM-5 will be excluded. These criteria are described in detail in the informed consent and assent forms (see Additional File $4 \mathrm{a}$ and $4 \mathrm{~b}$ ). If TD adolescents are excluded from the study due to any of the previously described exclusion criteria, they and their parents are encouraged to contact the clinicians associated with this study to discuss their concerns, if any.

Recruitment of adolescents with ASD will take place in the region of Leuven (Belgium) through the Leuven Autism Expertise Centre-a supraregional outpatient clinic supervised by Prof. Dr. Steyaert, supporting PI of this study-and the Leuven Autism Research Group (LAuRes, all authors are members. In addition, several contacts have been established with special education schools, autism advocacy organizations and support groups (f.i. VVA, Participate, "Autisme Centraal") as well as with independent clinical practices in and around the region of Leuven. TD adolescents will be recruited in the same region by contacting schools.

\section{Sample size}

Sample size was calculated for the cross-sectional phase using the G*Power tool [34] with a medium effect size ( $g$ $=0.59$ ) as reported in the meta-analysis of Cheng et al. [20], where baseline RSA values were compared between individuals with and without ASD. An a priori power analysis $(\alpha=0.05 ; 1-\beta=0.80)$ in a two-independent means design resulted in a total sample size of 74 adolescents, including 37 adolescents with ASD and 37 TD adolescents. In order to achieve an equal gender distribution, the sample size is further increased to 38 adolescents with ASD and 38 TD adolescents.

For the longitudinal phase, there was no evidence for an effect size from a representative study. Therefore, we consider a medium effect size $(d=0.50)$ to be representative of a clinically meaningful change. Comparison for the intervention phase was based on the supervised intervention group versus the sham-control group, resulting in an a priori power analysis $(\alpha=0.05 ; 1-\beta=0.80)$ for a two-independent means design. The total sample size resulted in a total of 128 adolescents with ASD, considering a hypothetical 20\% drop-out rate [35] and an equal gender distribution across both groups $(n=64 /$ group). Since the intervention during the follow-up phase (T1-T2) is a feasibility study, a sample containing $50 \%$ of the total sample was deemed to be sufficient in order to obtain reliable information regarding feasibility and acceptability concerning the non-supervised intervention. 


\section{Assessment}

As presented in Table 1, informed consent/assent and screening for eligibility will be performed first. In addition, participating adolescents and their parents will be asked to complete the questionnaires prior to scheduling the first study visit (assessment session T0).

In each assessment session (T0, T1, T2), a standardized stress-provoking protocol of $30 \mathrm{~min}$ will be executed in which physiological data will be continuously gathered (see the "Outcome measures" section). Furthermore, saliva samples will be collected at three different time points during each of the assessment sessions (see section 'Outcome Measures'). All behavioral data will be collected before the assessment sessions take place, as visualized in Fig. 1, except for the "visual analog scale for perceived stress," for which data will be gathered after the stress-provoking protocol. During this stressprovoking protocol, the participants will be asked to sit with their knees in a $90^{\circ}$ angle, with their hands on their thighs, palms facing upwards and eyes closed (during the first $10 \mathrm{~min}$ of baseline measurement only). To prevent temperature transference of the thighs towards the temperature sensor of the hands, a towel will be placed on the thighs [36]. Each of the assessments will be executed in the afternoon, as recommended by Hollocks et al. [37], to rule out influence of the cortisol awakening response as this can cause misleading results. In addition, participants will be asked to refrain from eating, intense physical activity, smoking, and drinking caffeine or alcoholic substances for at least one hour before testing [38]. Following the recommendations of Schmalenberger et al. [39], female participants will be asked whether they use contraceptives and when their last menstrual period was as these factors influence cardiac vagal activity.

During the first $10 \mathrm{~min}$ of the stress-provoking protocol, referred to as the "baseline period," participants are asked to sit quietly with their eyes closed as mentioned earlier. Subsequently, the "Stroop Word-Color Interference task" [40] and the "Social Stress Recall Task" (SSRT [2, 41];) will be used as stress-provoking tasks, each followed by a 5 -min resting period. Both tasks are validated to provoke stress reactivity as assessed in individuals with ASD [2, 42]. In total, the stress-provoking protocol consists of 5 periods (baseline, Stroop WordColor Interference Task, resting period, SSRT, resting period).

The first task in the stress-provoking protocol is the "Stroop Word-Color Interference task." A protocol previously used in children with ASD will be used as guidance [42]. Participants will perform a computerized version with five 1-min trials. During the first and the fifth trial, the presentation of words corresponding to color names are printed in the same color as the word itself. The remaining trials consist of incongruent stimuli, meaning that the color name is printed in another color than the word itself. The participants only need to name the color of the print. In addition, the time of the word presentation varies per trial between 1 and $2 \mathrm{~s}$. Prior to starting the task, participants are provided with ten practice stimuli to ensure understanding of the task.

The second task is the "Social Stress Recall Task." This task contains a 5-min stressor phase during which the participant is asked to describe the three most challenging aspects of his/her life on a day-to-day basis. The researcher can use prompts in order to guide the participant such as: "Can you tell me more about how that experience made you feel?" or "Can you remember what you did during that situation?" The timing of prompts will be recorded [2].

\section{Intervention}

The intervention is based on the biofeedback protocol provided by Lehrer et al [43]. and aims at increasing cardiac vagal modulation in adolescents with ASD as has been demonstrated in previous research [23].

In order to follow autism-specific treatment guidelines, all training sessions will be summarized and depicted in schedules [44]. This way, the different phases are predictable and the participants are able to follow each step by themselves. Participants will be asked to provide weekly updates on medication status by visit (during the supervised sessions) or by a reminder mail send by the researcher (during the follow-up phase).

To enhance treatment compliance and gain insight into the feasibility, the participants will be asked-via a secure web-based application (m-Path, KU Leuven)-to rate their level of comfort during the training on a scale using five smiley faces ranging between frustrated and satisfied after each training-session at home.

\section{Supervised RSA biofeedback}

This intervention part lasts for 5 weeks and contains the fixed scheme of one guided session per week with an experienced researcher (AT) and home practice on days without a guided session. Participants will be asked to breathe at their resonance frequency, which refers to the breathing frequency at which heart rate and the breathing pattern are in phase, thus maximizing RSA [23]. During the first session of this intervention, the resonance frequency of the participants will be determined using Biotrace+ Software (Mind Media B.V., www. mindmedia.com), based on the recommendations of Shaffer and Meehan [45]. Subsequently, the participants will be instructed to breathe at this frequency through stepwise guidance from the researcher using appropriate breathing techniques (with pursed lips and abdominal breathing) and the provision of a breathing pacer. 
During home practice, the participants will be provided with a breathing pacer application, individually set to practice at the specified resonance breathing frequency $[46,47]$. From the third supervised session onwards, participants will be instructed to breathe in phase with their heart rate. The latter will be visualized on a computer screen and will replace the breathing pacer installed at their resonance frequency as described for the previous sessions. However, they will continue breathing at resonance frequency during home practice. The sham-control group will follow the same steps as outlined above but they will practice at a normal breathing pace as determined during the baseline measurement at $\mathrm{T} 0$, thus faster than their resonance frequency. In addition, they will not receive any information regarding their own heart rate, as a default mode will be shown during the guided sessions. Each guided session will last approximately 30 min with several breaks in between whereas home practice sessions will last $20 \mathrm{~min}$, which will be divided into four blocks of $5 \mathrm{~min}$. The participants will be asked-via a secure web-based application (m-Path, KU Leuven) to rate the level of comfort during home practice on a scale using five smiley faces ranging between frustrated and satisfied after each session at home.

\section{Non-supervised RSA biofeedback: feasibility}

The non-supervised intervention will be home-based with one guided session at the start during which the individual resonance frequency of the participants will be determined and the procedure will be explained. Next, participants will breathe at this resonance frequency on a daily basis at home in four blocks of 5 min using a biofeedback smartphone application (Elite HRV, Asheville, North Carolina, USA). In addition, a compatible heart rate sensor (Polar ${ }^{\circ}$ H10, Polar, Finland) will monitor the participant's heart rate. Information regarding this monitoring will be sent to the researcher (AT). A secure webbased application will ask the participants to rate their level of comfort on a daily basis, as described previously for the supervised intervention.

\section{Randomization}

A computer-generated list will be used including block randomization procedures with a sequential allocation ratio of 1:1 for the supervised intervention group versus the sham-control intervention (T0-T1) and for the nonsupervised intervention group versus the no-intervention control group (T1-T2), based on the order of their enrolment. In addition, a stratification procedure based on gender and severity of ASD will be applied to obtain an equal division of these characteristics across the groups. The severity of ASD will be determined by the SRS-2, consisting of three groups to categorize the participants based on their norm-based $t$-scores (within a population with ASD). The severity levels are described as follows: $t$-score below 40 is categorized as mild, $t$-score between 41 and 60 is categorized as average, and $t$-score above 61 is categorized as severe. Randomization procedures will be performed by AT. Blinding of participants concerning group allocation is only feasible during the supervised intervention, not during the feasibility study with regard to the non-supervised intervention (T1-T2). After completion of the study, participants will be unblinded for group allocation upon request as described in the informed consent/assent.

\section{Outcome measures \\ Primary outcome measure}

Cardiac vagal modulation will be based on RSA, which can be calculated using a time domain measure (Root Mean Square of Successive Differences between normal heartbeats, RMSSD) and a frequency domain measure (Logarithm of High-Frequency Heart Rate Variability (LnHF-HRV)) [36]. For these parameters, 3-lead electrocardiographic recordings of heart rate (chest placement) will be performed using a NeXus-10 MKII biofeedback device and Biotrace+ Software (Mind Media B.V., www. mindmedia.com) (see subsequent section "Physiological parameters"). The recommendations of the Task Force of the European Society of Cardiology and the North American Society of Pacing and Electrophysiology [48] and Laborde et al. [36] regarding the calculation of this outcome measure will be followed.

\section{Secondary outcome measures}

Physiological parameters Heart rate, breathing frequency, skin conductance, and fingertip temperature. The NeXus-10 MKII biofeedback device and Biotrace+ Software (Mind Media B.V., www.mindmedia.com) will be used to register the physiological parameters. Continuous measurement during the stress-provoking assessment will be performed with multiple electrodes placed on the body including:

A 3-lead ECG on the chest with disposable pre-gelled electrodes (Kendall ${ }^{\text {tw }}$ ECG Electrodes Arbo $^{\text {Tw }}$ H124SG, Covidien, Ireland) without specific skin preparations for the recording of heart rate at a sampling rate of 256 SPS.

An elastic band with stretch-sensitive sensors around the waist for the recording of the breathing frequency at a sampling rate of 32 SPS.

Two electrodes for the measurement of skin conductance with a sampling rate of 32 SPS strapped around the palmar side of the distal phalanx of the index and ring finger on the non-dominant hand.

One temperature sensor with a sampling rate of 32 SPS placed around the palmar side of the distal phalanx 
of the middle finger on the non-dominant hand for the recording of fingertip temperature.

Placement of the electrodes is visualized in Fig. 2.

Cortisol measurement Saliva samples will be collected to determine the level of cortisol at three time points during each assessment. The first one (baseline) is collected before the start of the stress-provoking protocol. The second and the third samples are collected at 35 and $45 \mathrm{~min}$ after baseline, respectively. The timing of the second and the third cortisol sample corresponds to a time-laps of $20 \mathrm{~min}$ after each of the stress-provoking tasks. Thus, the second sample reflects the cortisol level for the Stroop Word-Color Interference Task while the third corresponds to the cortisol level for the SSRT. Salivette $^{\oplus}$ Cortisol cotton swabs (Sarstedt Inc., Rommelsdorf, Germany) will be used. These swabs are validated for use with salivary cortisol. After collection, the swabs will be stored under appropriate conditions $\left(-20^{\circ} \mathrm{C}\right)$ at the biobank of the KU Leuven and analyzed in a collaborative lab from the KU Leuven.

Behavioral data Autism Quotient - Adolescent Version: The AQ [33] identifies the extent of autistic traits shown by a person of normal intelligence based on parentreport. A higher total score reflects a higher presentation of autism-related symptoms. A cut-off of 32 or higher is used for the exclusion of TD adolescents.

Social Responsiveness Scale - Second edition: The SRS2 [49] is a quantitative assessment based on parentreport. This questionnaire identifies social impairment associated with ASD and quantifies its severity by using five subscales (Social Awareness, Social Cognition, Social Communication, Social Motivation, and Restricted
Interests and Repetitive Behavior). Higher scores reflect a higher level of impairment.

Repetitive Behavior Scale - Revised: The RBS-R [50] is used to score the number and intensity of repetitive and restrictive behaviors based on parent-report. A higher total score indicates a higher prevalence of repetitive and restrictive behaviors. In addition, these behaviors are clustered in six categories: Stereotyped Behavior, Self-injurious Behavior, Compulsive Behavior, Ritualistic Behavior, and Sameness Behavior. Parents are also asked to rate the impact of these behaviors on daily functioning on a scale from 0 (meaning no interference with daily life) to 100 (extremely problematic related to functioning in daily life).

Strengths and Difficulties Questionnaire: The SDQ [5153 ] is a brief behavioral screen resulting in five categories of behavioral problems: Emotional problems, Conduct problems, Hyperactivity, Peer problems, and Prosocial behavior. Higher total scores-excluding the prosocial category-indicate more behavioral problems. Both the self-report and parent-report versions will be used.

Perceived Stress Scale - Adolescent Version: The PSS [54] measures the degree to which situations in the adolescent's life are appraised as stressful. This measure is based on adolescent self-report in which items are adapted according to the specific problems that adolescents can encounter in their lives, such as school-related stress. A higher total score is related to higher perceived stress levels.

Depression Anxiety and Stress Scale - 21-item version: The DASS-21 [55] measures the presence of negative emotions based on adolescent self-report. Separate scale scores are calculated for symptoms related to depression, anxiety, and stress with higher scores reflecting a higher prevalence of the related symptoms.

Physical Activity Vital Sign: The PAVS [56] determines the level of physical activity during the last week based
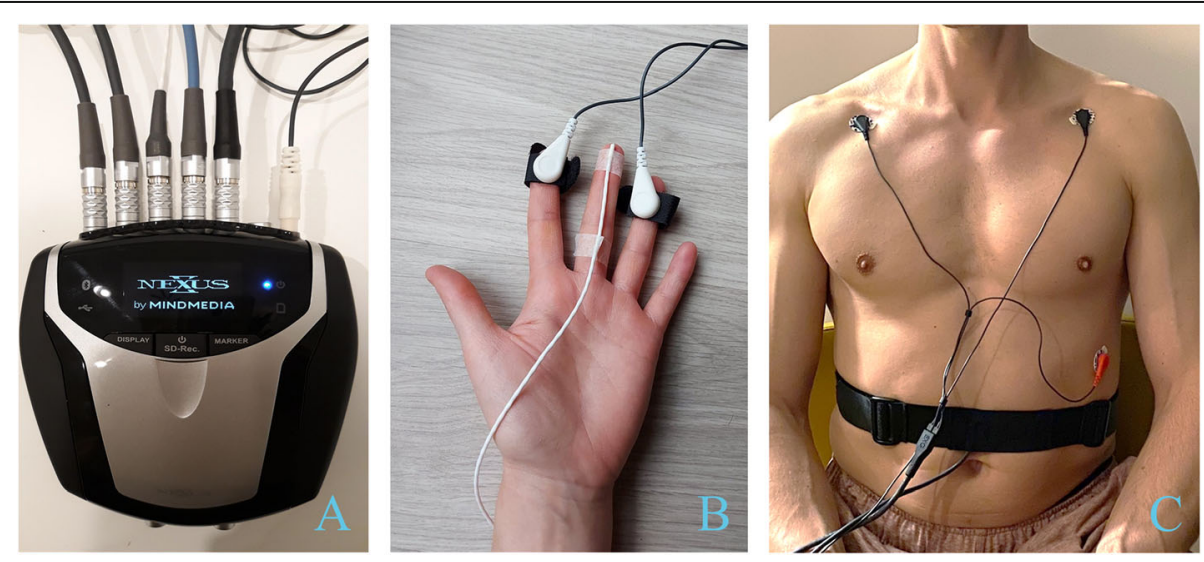

Fig. 2 Overview placement of physiological sensors. Set-up of the biofeedback system. A NeXus-10 MKII device. B Sensors for hand temperature $(n=1)$ and skin conductance $(n=2)$. C Sensors concerning heart rate $(n=3)$ and breathing frequency $(n=1)$ 
on adolescent self-report. This measure consists of two short questions related to [1] the number of days the adolescent was physically active and [2] the number of minutes (on average) this physical activity was endured.

Sensory hypersensitivity based on a visual analog scale: Measures the self-reported influence of sensory hyperresponsivity on a visual analog scale going from 0 (meaning no perceived sensory hyper-responsivity) to 10 (meaning presence of severe sensory hyper-responsivity and negative impact on functioning).

Perceived stress based on a visual analog scale: To rate the self-reported perceived stress level on a visual analog scale per stressor after the stress-provoking protocol. The scale score can vary between 0 (meaning no stress) and 10 (meaning the highest level of perceived stress possible).

Data handling and statistical analysis For all data, group-based means and standard deviations will be reported for each of the assessment sessions as descriptive data and will be used for statistical analyses.

The raw scores of the behavioral data will be gathered via a Qualtrics and m-Path account protected by the KU Leuven. Calculation and further analysis of the group-based means (TD versus ASD and intervention versus control) for each of the assessment sessions (T0 alone and $\mathrm{T} 0, \mathrm{~T} 1$, and $\mathrm{T} 2$ ) will be performed using SPSS (IBM SPSS Statistics, version 27). In addition, analysis and comparison of cortisollevels between groups (TD versus ASD and intervention versus control) for the effects of each period (baseline, Stroop Word-Color Interference Task and SSRT) in each of the assessment sessions (T0 alone and T0, T1, and T2) will be performed with SPSS as well. Physiological data regarding heart rate will be exported from BioTrace+ as raw data of the five periods per assessment session into IBI text files for each participant. These will be exported to Kubios HRV Premium (version 3.4.3, University of Eastern Finland, Kuopio, Finland), which will be used to clean the data using the automatic artifact correction algorithm included in the software. Subsequently, analysis of the data regarding heart rate variability will be performed to determine cardiac vagal modulation expressed as mean values of RMSSD and LnHF-HRV, as described in the "Primary outcome measure" section. A combination of researcher-developed scripts in MATLAB R2020b (MathWorks, Natick, Massachusetts, USA) and SPSS (IBM SPSS Statistics, version 27) will perform preprocessing and analysis of the mean values of skin conductance, fingertip temperature, and respiration for the five separate periods in each of the assessment sessions of the participants.
One member of the research team (AT) will gather all data to conduct statistical analyses using MATLAB R2020b statistical packages and SPSS version 27 to test the main hypotheses. For all analyses, the level of significance is set at 0.05 with appropriate corrections for related post hoc analysis. In addition, the strategy of intention-to-treat will be applied. If normal distribution of physiological data is not present, log-transformation will be conducted as recommended by Laborde et al. [36]. For participants with missing data on any measure, multiple imputation or an ignorability analysis based on the likelihood will be used. If available, reasons for dropout will be listed in order to gain more insight into the feasibility of both interventions.

Cross-sectional data comparison: Data from each period of the stress-provoking protocol at T0 will be used to compare the adolescents with ASD $(n=38)$ with their TD peers $(n=38)$ in a repeated measures ANOVA with group (ASD vs TD) as between-group factor. Pearson correlation analysis will be performed to determine correlations between the primary outcome measure and secondary outcome measures.

Efficacy of the RSA biofeedback intervention; The efficacy of both interventions will be determined by intention-to-treat analysis using physiological data (from each period of the stress-provoking protocol) and cortisol data in a mixed model analysis with 'group' (supervised intervention versus non-supervised intervention versus sham versus no intervention) as a between-group factor and 'time' (T0, T1, and T2) as a within-group factor.

Change in behavioral outcome measures after supervised RSA biofeedback: In order to determine the influence of the intervention on the behavioral outcome measures, pre-to-post difference scores (T0-T2) will be calculated for the primary outcome measure and the behavioral outcome measures. These differences will be used in a correlation analysis to determine whether positive effects on the primary outcome measure correlate with similar effects on the behavioral parameters.

Impact of behavioral outcome measures on treatment efficacy: Correlation analyses will be performed between baseline behavioral parameters and the pre-to-post difference scores (T0-T2) for the primary outcome measure. The behavioral parameters with the largest correlation with the primary outcome measure will be used as a covariate or additional between-subject factor in the mixed model analyses to determine the influence of baseline behavioral characteristics of the participants on the efficacy of the biofeedback interventions.

\section{Discussion}

The results of the cross-sectional phase will provide more insight into the differences of autonomic 
functioning between adolescents with ASD and their TD peers. In addition, the association between autonomic functioning and behavioral measures will be clarified. The longitudinal phase will determine the efficacy of a supervised RSA biofeedback intervention in adolescents with ASD. This intervention may provide multiple benefits for this population specifically due to its possibility to be executed at home, as will be examined during the feasibility study of the non-supervised RSA-based biofeedback intervention in this study. The latter can facilitate the integration and transfer into their daily routine. Given the high rate of co-occuring psychological symptoms and a hypothesized association between lower cardiac vagal modulation and these symptoms in individuals with ASD, an upregulation of cardiac vagal modulation may be valuable for this particular population. This can be accomplished by RSA-based biofeedback intervention, as has been previously demonstrated in other populations including physical, behavioral, and cognitive conditions [22-30].

\section{Trial status}

This study was registered at ClinicalTrials.gov on November 13, 2020 (NCT04628715). At the time of initial manuscript submission, recruitment had started (November 26,2020$)$ but not yet completed.

\section{Abbreviations}

ASD: Autism spectrum disorder; AQ: Autism quotient; DASS: Depression Anxiety and Stress Scales; DSM-IVN: Diagnostics and Statistical Manual of mental disorders; ECG: Electrocardiogram; FU: Follow-up; HF-HRV: HighFrequency Heart Rate Variability; HRV: Heart rate variability; PAVS: Physical activity vital sign; PSS: Perceived Stress Scale; RBS-R: Repetitive Behavior Scale - Revised; RMSSD: Root mean square of successive differences; RSA: Respiratory sinus arrhythmia; SDQ: Strengths and Difficulties Questionnaire; SRS: Social Responsiveness Scale; SSRT: Social Stress Recall Task; TD: Typically developing; VAS: Visual analog scale

\section{Supplementary Information}

The online version contains supplementary material available at https://doi. org/10.1186/s13063-021-05709-4.

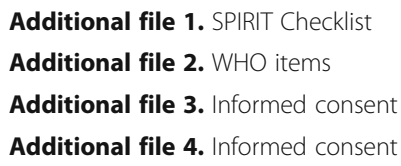

\section{Acknowledgements}

Not applicable.

\section{Authors' contributions}

All authors contributed to the writing of this manuscript and the grant proposal. AT leads the study and data management under the supervision of TVD, who provided facilities and equipment. All authors read and approved the final manuscript.

\section{Funding}

This work is supported by the Marguerite-Marie Delacroix foundation with grant number GV/B-363, awarded to AT. The funder will not have any role in any part of this study.

\section{Availability of data and materials}

All data from the participants will be de-identified and provided with a unique code. The coded data will be stored for 20 years in secured databases of the Research Group for Adapted Physical Activity and Psychomotor Rehabilitation, protected by the KU Leuven, and will only be accessible by researchers of collaborating labs. The key to the coded data will be stored securely and confidentially in a separate electronic file. The saliva samples will be stored under appropriate conditions during the study at the biobank of the KU Leuven and will be destroyed afterwards. Participants waive any intellectual property rights on findings that might result from the analysis of their saliva samples.

\section{Declarations}

Ethics approval and consent to participate

Ethical approval for this study was granted by both the Ethics Committee UPC KU Leuven on July 2, 2020 (ref. EC2020-541, version 1.0) and the Ethics Committee Research UZ/KU Leuven on October 20, 2020 (ref: S64219, version 2.0) and on April 9, 2021 (ref: S64219, version 3.0, amendment). A copy of the original approval and its translation has been added as "Additional File $1 \mathrm{a}, 1 \mathrm{~b}, 1 \mathrm{c}$ and $1 \mathrm{~d}$." The researchers will permit study-related monitoring, audits, Ethical Committee review and regulatory inspection, providing direct access to all related source data/documents. Every substantial adjustment to the protocol will be communicated to the Ethics Committee UPC KU Leuven and the Ethics Committee Research UZ/KU Leuven as an amendment to the protocol. Only after approval of this amendment, the adjustments can be implemented and communicated to the researchers and participants. Parents of the participants should provide informed consent in addition to the informed assent provided by the participants themselves. The informed consent and assent forms will be gathered by AT. Participants may withdraw consent and participation at any time. The participant's request to withdraw from the study will always be respected and reasons to withdraw are not obliged to be mentioned. The sponsor of this study is KU Leuven (Oude Markt 13, 3000 Leuven, Belgium) and will have no role in any part of this study. Translated versions have been added as "Additional File 4a and 4b."

\section{Consent for publication}

Authorship to publications will be determined in accordance with the requirements published by the International Committee of Medical Journal Editors and in accordance with the requirements of the respective peerreviewed medical journal. There will also be a general dissemination of the study results for the participants and personal results will be provided upon request.

\section{Competing interests}

The authors declare that they have no competing interests.

\section{Author details}

'Department of Rehabilitation Sciences, Research Group for Adapted Physical Activity and Psychomotor Rehabilitation, KU Leuven, Leuven, Belgium.

${ }^{2}$ Leuven Autism Research (LAuRes), KU Leuven, Leuven, Belgium.

${ }^{3}$ Department of Child Psychiatry, UPC KU Leuven, Leuven, Belgium.

${ }^{4}$ Department of Rehabilitation Sciences, Neuromotor Rehabilitation Research Group, KU Leuven, Leuven, Belgium.

Received: 29 April 2021 Accepted: 8 October 2021

Published online: 21 October 2021

References

1. American Psychiatric Association. Diagnostic and statistical manual of mental disorders. 5th ed. Washington DC: American Psychiatric Association; 2013

2. Bishop-Fitzpatrick L, Minshew NJ, Mazefsky CA, Eack SM. Perception of life as stressful, not biological response to stress, is associated with greater social disability in adults with autism spectrum disorder. J Autism Dev Disord. 2017;47(1):1-16. https://doi.org/10.1007/s10803-016-2910-6.

3. Groden J, Baron MG, Groden G. Assessment and coping strategies. In: Baron MG, Groden J, Groden G, Lipsitt L, editors. Stress and coping in autism. New York: Oxford Univeristy Press, Inc; 2006. p. 15-41.

4. Corbett BA, Simon D. Adolescence, stress and cortisol in autism spectrum disorders. OA Autism. 2014;1(1):2. 
5. Benevides TW, Lane SJ. A review of cardiac autonomic measures: considerations for examination of physiological response in children with autism spectrum disorder. J Autism Dev Disord. 2015;45(2):560-75. https:// doi.org/10.1007/s10803-013-1971-z

6. Condy EE, Scarpa A, Friedman BH. Restricted repetitive behaviors in autism spectrum disorder: a systematic review from the neurovisceral integration perspective. Biol Psychol. 2019;148:107739. https://doi.org/10.1016/j. biopsycho.2019.107739.

7. Ellenbroek BA, Sengul HK. Autism spectrum disorders: autonomic alterations with a special focus on the heart. Heart and Mind. 2017:1(2):78. https://doi. org/10.4103/hm.hm_5_17.

8. Lydon S, Healy O, Reed P, Mulhern T, Hughes BM, Goodwin MS. A systematic review of physiological reactivity to stimuli in autism. Dev neurorehabil. 2016 19(6):335-55. https://doi.org/10.3109/17518423.2014.971975.

9. Patriquin MA, Hartwig EM, Friedman BH, Porges SW, Scarpa A. Autonomic response in autism spectrum disorder: relationship to social and cognitive functioning. Biol Psychol. 2019;145:185-97. https://doi.org/10.1016/j. biopsycho.2019.05.004.

10. Shaffer F, McCraty R, Zerr CL. A healthy heart is not a metronome: an integrative review of the heart's anatomy and heart rate variability. Frontiers in psychology. 2014. https://doi.org/10.3389/fpsyg.2014.01040.

11. Shaffer F, Ginsberg JP. An overview of heart rate variability metrics and norms. Front Public Health. 2017;5. https://doi.org/10.3389/fpubh.2017.00258.

12. Beauchaine TP, Bell Z, Knapton E, McDonough-Caplan H, Shader,T, Zisner A. Respiratory sinus arrhythmia reactivity across empirically based structural dimensions of psychopathology: a meta-analysis. Psychophysiology. 2019; doi:https://doi.org/10.1111/psyp.13329, Respiratory sinus arrhythmia reactivity across empirically based structural dimensions of psychopathology: A meta-analysis, 56, 5, e13329.

13. Fiskum C. Psychotherapy beyond all the words: Dyadic expansion, vagal regulation, and biofeedback in psychotherapy. Journal of Psychotherapy Integration. 2019;29(4):412-25. https://doi.org/10.1037/int0000174.

14. Beauchaine TP. Respiratory Sinus Arrhythmia: A Transdiagnostic Biomarker of Emotion Dysregulation and Psychopathology. Curr Opin Psychol. 2015;3: 43-7. https://doi.org/10.1016/j.copsyc.2015.01.01.

15. Graziano P, Derefinko K. Cardiac vagal control and children's adaptive functioning: a meta-analysis. Biol Psychol. 2013;94(1):22-37. https://doi.org/1 0.1016/j.biopsycho.2013.04.011

16. Patriquin MA, Lorenzi J, Scarpa A, Calkins SD, Bell MA. Broad implications for respiratory sinus arrhythmia development: associations with childhood symptoms of psychopathology in a community sample. Dev Psychobiol. 2015:57(1):120-30. https://doi.org/10.1002/dev.21269.

17. Porges SW. The Polyvagal Theory: Neurophysiological Foundations of Emotions, Attachment, Communication, Self-Regulation. New York: W.W. Norton \& Company; 2011.

18. Muscatello RA, Vandekar SN, Corbett BA. Evidence for decreased parasympathetic response to a novel peer interaction in older children with autism spectrum disorder: a case-control study. J Neurodev Disord. 2021; 13(1):6. https://doi.org/10.1186/s11689-020-09354-x.

19. Corbett BA, Muscatello RA, Baldinger C. Comparing stress and arousal systems in response to different social contexts in children with ASD. Biol Psychol. 2019;140:119-30. https://doi.org/10.1016/j.biopsycho.2018.12.010.

20. Cheng $Y-C$, Huang $Y-C$, Huang W-L. Heart rate variability in individuals with autism spectrum disorders: a meta-analysis. Neurosci Biobehav Rev. 2020; 118:463-71. https://doi.org/10.1016/j.neubiorev.2020.08.007.

21. Lory C, Kadlaskar G, McNally Keehn R, Francis AL, Keehn B. Brief report: reduced heart rate variability in children with autism spectrum disorder. J Autism Dev Disord. 2020;50(11):4183-90. https://doi.org/10.1007/s10803-02 0-04458-8.

22. Chudleigh C, Savage B, Cruz C, Lim M, McClure G, Palmer DM, et al. Use of respiratory rates and heart rate variability in the assessment and treatment of children and adolescents with functional somatic symptoms. Clin Child Psychol Psychiatry. 2019;24(1):29-39. https://doi.org/10.1177/135910451 8807742.

23. Lehrer P, Kaur K, Sharma A, Shah K, Huseby R, Bhavsar J, et al. Heart rate variability biofeedback improves emotional and physical health and performance: a systematic review and meta analysis. Appl Psychophysiol Biofeedback. 2020;45(3):109-29. https://doi.org/10.1007/s10484-020-09466-z.

24. Darling KE, Benore ER, Webster EE. Biofeedback in pediatric populations: a systematic review and meta-analysis of treatment outcomes. Transl Behav Med. 2019. https://doi.org/10.1093/tbm/ibz124.
25. Gevirtz R. The promise of heart rate variability biofeedback: evidence-based applications. Biofeedback. 2013:41(3):110-20. https://doi.org/10.5298/10815937-41.3.01.

26. Kennedy L, Parker SH. Biofeedback as a stress management tool: a systematic review. Technology \& Work: Cognition; 2019. https://doi.org/10.1 007/s10111-018-0487-x, Biofeedback as a stress management tool: a systematic review

27. Knox M, Lentini J, Cummings T, McGrady A, Whearty K, Sancrant L. Gamebased biofeedback for paediatric anxiety and depression. Ment health in fam med. 2011:8:3.

28. Lehrer PM, Gevirtz R. Heart rate variability biofeedback: how and why does it work? Frontiers in Psychology. 2014;5. https://doi.org/10.3389/fpsyg.2014. 00756.

29. Thabrew H, Ruppeldt P, Sollers JJ 3rd. Systematic review of biofeedback interventions for addressing anxiety and depression in children and adolescents with long-term physical conditions. Appl Psychophysiol Biofeedback. 2018;43(3):179-92. https://doi.org/10.1007/s10484-018-9399-z.

30. Goodman MS, Castro N, Sloan M, Sharma R, Widdowson M, Herrera E, et al. A neurovisceral approach to autism: targeting self-regulation and core symptoms using neurofeedback and biofeedback. NeuroRegulation. 2018. https://doi.org/10.15540/nr.5.1.9.

31. Chan A-W, Tetzlaff JM, Gøtzsche PC, Altman DG, Mann H, Berlin JA, et al. SPIRIT 2013 explanation and elaboration: guidance for protocols of clinical trials. BMJ: British Medical Journal. 2013;346(jan08 15). https://doi.org/10.113 6/bmj.e7586.

32. American Psychiatric Association. Diagnostic and statistical manual of mental disorders. 4, text rev ed 2000.

33. Baron-Cohen S, Hoekstra RA, Knickmeyer R, Wheelwright S. The autismspectrum quotient (AQ) - adolescent version. J Autism Dev Disord. 2006 36(3):343-50. https://doi.org/10.1007/s10803-006-0073-6.

34. Faul FEE, Buchner A, Lang A-G. Statistical power analyses using G*Power 3.1 Tests for correlation and regression analyses. Behav Res Methods. 2009. https://doi.org/10.3758/BRM.41.4.1149.

35. Furlan AD, Pennick V, Bombardier C, van Tulder M. updated method guidelines for systematic reviews in the Cochrane Back Review Group. Spine (Phila Pa 1976). 2009. 2009:34(18):1929-41. https://doi.org/10.1097/BRS. 0b013e3181b1c99f.

36. Laborde S, Mosley E, Thayer JF. Heart rate variability and cardiac vagal tone in psychophysiological research - recommendations for experiment planning, data analysis, and data reporting. frontiers in psychology. 2017; doi:https://doi.org/10.3389/fpsyg.2017.00213.

37. Hollocks MJ, Howlin P, Papadopoulos AS, Khondoker M, Simonoff E. Differences in HPA-axis and heart rate responsiveness to psychosocial stress in children with autism spectrum disorders with and without co-morbid anxiety. Psychoneuroendocrinology. 2014;46:32-45. https://doi.org/10.1016/j. psyneuen.2014.04.004

38. Smeekens I, Didden R, Verhoeven EW. Exploring the relationship of autonomic and endocrine activity with social functioning in adults with autism spectrum disorders. J Autism Dev Disord. 2015;45(2):495-505. https:// doi.org/10.1007/s10803-013-1947-z.

39. Schmalenberger KM, Eisenlohr-Moul TA, Wurth L, Schneider E, Thayer JF, Ditzen $B$, et al. A systematic review and meta-analysis of within-person changes in cardiac vagal activity across the menstrual cycle: implications for female health and future studies. Journal of clinical medicine. 2019;8(11). https://doi.org/10.3390/jcm8111946.

40. Stroop JR. Studies of interference in serial verbal reactions. J Exp Psychol Gen. 1935;18(6):643-62. https://doi.org/10.1037/h0054651.

41. Richman L, Bennett G, Pek J, Siegler I, Williams R. Discrimination, dispositions, and cardiovascular responses to stress. Health Psychol. 2007; 26(6):675-83. https://doi.org/10.1037/0278-6133.26.6.675.

42. Kushki A, Drumm E, Pla Mobarak M, Tanel N, Dupuis A, Chau T, et al. Investigating the autonomic nervous system response to anxiety in children with autism spectrum disorders. PLoS One. 2013;8(4):e59730. https://doi. org/10.1371/journal.pone.0059730.

43. Lehrer P, Vaschillo B, Zucker T, Graves J, Katsamanis M, Aviles M, et al. Protocol for heart rate variability biofeedback training. Biofeedback. 2013; 41(3):98-109. https://doi.org/10.5298/1081-5937-41.3.08.

44. Fuentes J, Hervás A, Howlin P. ESCAP practice guidance for autism: a summary of evidence-based recommendations for diagnosis and treatment. Eur Child Adolesc Psychiatry. 2021;30(6):961-84. https://doi.org/10.1007/ s00787-020-01587-4 
45. Shaffer F, Meehan ZM. A practical guide to resonance frequency assessment for heart rate variability biofeedback. Front. Neurosci. 2020;14. https://doi. org/10.3389/fnins.2020.570400.

46. Trex L. Paced Breathing [Mobile app]. Google Play Store; 2015.

47. Awesome Labs LLC. Awesome Breathing: Pacer Timer [Mobile App]. App Store; 2020.

48. Malik M. Heart rate variability: Standards of measurement, physiological interpretation, and clinical use: Task force of the European Society of Cardiology and the North American Society for Pacing and Electrophysiology. Ann Noninvasive Electrocardiol. 1996;1 (2):151-81. https:// doi.org/10.1111/j.1542-474X.1996.tb00275.X.

49. Constantino JN, Gruber CP. Social Responsiveness Scale-Second Edition (SRS-2). CA: Western Psychological Services: Torrance; 2012

50. Bodfish JW, Symons FJ, Parker DE, Lewis MH. Varieties of repetitive behavior in autism: Comparisons to mental retardation. J Autism Dev Disord. 2000; 30(3):237-43. https://doi.org/10.1023/a:1005596502855.

51. Goodman R, Meltzer H, Bailey V. The Strengths and Difficulties Questionnaire: a pilot study on the validity of the self-report version. Eur Child Adolesc Psychiatry. 1998;7(3):125-30. https://doi.org/10.1007/ s007870050057.

52. van Widenfelt BM, Goedhart AW, Treffers PD, Goodman R. Dutch version of the Strengths and Difficulties Questionnaire (SDQ). Eur Child Adolesc Psychiatry. 2003;12(6):281-9. https://doi.org/10.1007/s00787-003-0341-3.

53. Goodman R. The Strengths and Difficulties Questionnaire: a research note. J Child Psychol Psychiatry. 1997;38(5):581-6. https://doi.org/10.1111/j.1469-761 0.1997.tb01545.x

54. Van der Ploeg J. Stress bij kinderen. Houten: Bohn Stafleu van Loghum, onderdeel van Springer Media BV; 2013. https://doi.org/10.1007/978-90-3680419-6.

55. de Beurs E, Van Dyck R, Marquenie LA, Lange A, Blonk RWB. De DASS: een vragenlijst voor het meten van depressie, angst en stress. Gedragstherapie. 2001;34.

56. Greenwood J, Joy E, Stanford J. The physical activity vital sign: a primary care tool to guide counseling for obesity. J Phys Act Health. 2010;7(5):571-6. https://doi.org/10.1123/jpah.7.5.571.

\section{Publisher's Note}

Springer Nature remains neutral with regard to jurisdictional claims in published maps and institutional affiliations.

Ready to submit your research? Choose BMC and benefit from:

- fast, convenient online submission

- thorough peer review by experienced researchers in your field

- rapid publication on acceptance

- support for research data, including large and complex data types

- gold Open Access which fosters wider collaboration and increased citations

- maximum visibility for your research: over $100 \mathrm{M}$ website views per year

At $\mathrm{BMC}$, research is always in progress.

Learn more biomedcentral.com/submissions 